\title{
Chemical and electromagnetic mechanisms of tip-enhanced Raman scattering
}

\author{
Mengtao Sun, ${ }^{*}$ Yurui Fang, ${ }^{a}$ Zhilin Yang ${ }^{a b}$ and Hongxing $\mathrm{Xu}^{a c}$ \\ Received 6th May 2009, Accepted 30th July 2009 \\ First published as an Advance Article on the web 27th August 2009 \\ DOI: $10.1039 / \mathbf{b} 909006 a$
}

In this paper, we attempt to reveal the nature of the chemical and electromagnetic mechanisms of tip-enhanced Raman scattering (TERS). Direct visual evidence regarding the chemical mechanism via charge transfer was obtained with charge difference density. It is found that there are several kinds of charge transfer: (1) tip to molecule, (2) surface to molecule, (3) tip and surface to molecule simultaneously, and (4) tunneling charge transfer between the tip and the surface. Direct evidence regarding the electromagnetic mechanism via intracluster (tip or surface) charge redistribution was also revealed via charge difference density. The distance (between tip and surface) dependence of the near electric field distribution and the TERS enhancement at different incident lights is also discussed using the three-dimensional finite-difference time-domain (FDTD) method. The electromagnetic enhancement of double-tip TERS is approximately 10 times larger than that of conventional TERS. Theoretical results reveal that plasmon coupling effects between the metal tip and surface play an important role in TERS.

\section{Introduction}

Surface-enhanced Raman scattering (SERS) is a well-known phenomenon where the Raman signal from adsorbates on a roughened metal surface is enhanced by factors as large as $10^{11}$, thereby making the detection of single molecules possible. These large enhancement factors arise from the excitation of surface plasmons (SPs) on roughened metals and/or from "first layer" enhancements which includes charge transfer between adsorbates and metals at resonance excitations. ${ }^{1,2}$ However, one of the most severe restrictions in the application of SERS to a wide variety of problems in ultrahigh vacuum single-crystal surface science, electrochemistry, heterogeneous catalysis, microelectronics, and tribology is the requirement that the surface be roughened or nanostructured $\mathrm{Ag}, \mathrm{Au}$, or $\mathrm{Cu}^{3}$

While it is based on the same phenomenon as SERS, tipenhanced Raman scattering (TERS) can circumvent these limitations and achieve complete substrate generality. ${ }^{4-8}$ When a metallic probe outfitted with a nanometric tip is illuminated with an optical field, conductive free electrons collectively oscillate at the surface of the metal. The electrons (and the positive charge) are concentrated at the tip apex and generate a strong external electric field. Photon energy is confined to the local vicinity of tip. Therefore, the metallic tip works as a photon reservoir. ${ }^{9}$ The advantages ${ }^{9}$ of TERS over SERS include: (1) TERS occurs on a single metallic nanotip which is capable of specifying an observation position for analysis with nanometric accuracy. (2) A nanotip is able to perturb

${ }^{a}$ Beijing National Laboratory for Condensed Matter Physics, Institute of Physics, Chinese Academy of Sciences, P. O. Box 603-146, Beijing, 100190, P. R. China.E-mail:mtsun@aphy.iphy.ac.cn

${ }^{b}$ Department of Physics, Xiamen University, Xiamen, 361005 ,

P. R. China

${ }^{c}$ Division of Solid State Physics, Lund University, Lund, 22100, Sweden molecules dynamically, electrically, or magnetically due to the fact that the tip is controlled by another type of scanning probe microscopy, e.g. atomic force microscopy or scanning tunneling microscopy. Zenobi and coworkers found that nanoscale roughness on metal surfaces can increase tip-enhanced Raman scattering by an order of magnitude. ${ }^{8}$ There are two kinds of TERS, as illustrated in Fig. 1. For conventional TERS the surface is flat ${ }^{5}$ while, for double-tip TERS, a fixed nanoprotrusion (i.e., a gold nanoparticle made using an aerosol technique) is placed on a flat gold surface. ${ }^{7}$ Comparing this to the case of a gold tip on a flat gold surface, Chen et al. found that a larger Raman enhancement can be obtained using the double-tip configuration. ${ }^{7}$

The mechanisms of electromagnetic (physical) enhancement and charge transfer (chemical) enhancement in SERS have been extensively investigated both experimentally and theoretically. ${ }^{1-8,10-14}$ Similarly, the physical enhancement effects (e.g. SPs) and chemical enhancement effects can be expected in TERS. ${ }^{9}$ The local field distribution near a metallic probe tip has been studied via simulations using the three-dimensional finite-difference time-domain (FDTD) method. ${ }^{15,16}$ These simulations show that a metallic probe localizes and strongly amplifies the optical field through the resonance effect of the plasmon at the probe tip. While the insight into the chemical mechanism on TERS was limited, it is very important to theoretically reveal the nature of the chemical enhancement on TERS.

The aim of this paper is to try to reveal the nature of the TERS chemical enhancement in a direct visual way. With charge difference density, ${ }^{17}$ we provide direct visual evidence for the chemical mechanism via charge transfer from tip (and/or surface) to molecule, and tunneling charge transfer between tip and surface. The direct evidence for the electromagnetic mechanism via intracluster (tip and/or surface) charge redistribution was also revealed with charge difference 


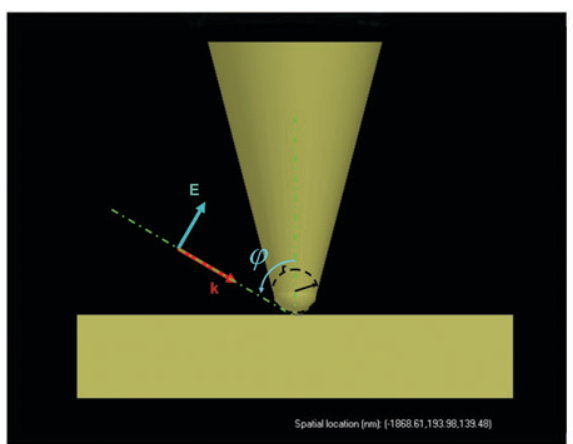

(a)

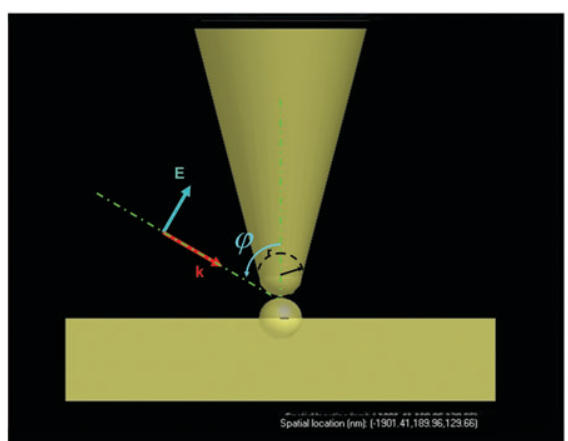

(b)

Fig. 1 (a) Illustration of a conventional TERS and (b) double-tip TERS in our calculations, where the radius of the final tip apex is $25 \mathrm{~nm}$.

density. We studied the effect of tip shape and size on TERS and tip-enhanced resonance Raman scattering (TERRS) using a quantum chemical method. Using the 3D-FDTD method, we also report the distance-dependent (between tip and surface) distribution of the near electric field enhancement for TERS at different incident lights. Finally, the electromagnetic enhancements of double-tip TERS are compared with those of the conventional TERS.

\section{Methods}

Recent photoelectron spectroscopy and relativistic density functional calculations strongly predict that $\mathrm{Au}_{20}$ has a tetrahedral geometry similar to a fragment of bulk face-centered cubic gold. ${ }^{18}$ In this paper, the tetrahedral $\mathrm{Au}_{20}$ configuration is adopted, which is a relaxed fragment of the face-centered cubic (fcc) lattice of bulk gold and is one of the local minima for the $\mathrm{Au}_{20}$ cluster. ${ }^{18}$ For tetrahedral $\mathrm{Au}_{20}$, there are two very different binding sites [S-complex and V-complex, see Fig. 2(a) and (b)]. The former consists of an on-top binding onto one of the four faces of the tetrahedral $\mathrm{Au}_{20}$ configuration, which represents a (111) surface of fcc silver, while the latter consists of binding onto one of its vertices, which represents an ad-atom site. It has been found that the calculated SERS spectrum using the S-complex is in better agreement with the experimental results (for much large nanoparticles) than that of the V-complex. ${ }^{13}$ In this paper, we use the S-complex in our SERS calculations. For the TERS calculations, an S-complex-tip $\left(\mathrm{Au}_{2}\right.$ and $\left.\mathrm{Au}_{20}\right)$ was employed. All of the models in Fig. 2(a), (c) and (d) were optimized with density functional theory (DFT), ${ }^{19}$ using the B3PW91 functional, ${ }^{20,21}$ LANL2DZ basis $\mathrm{set}^{22}$ for $\mathrm{Au}$, and the 6-31G basis set for $\mathrm{C}, \mathrm{N}$ and $\mathrm{H}$. Normal Raman scattering (NRS) of the SERS and TERS models were calculated with the same method at zero frequency. The electronic structures of these models were calculated using a time-dependent DFT (TD-DFT) method, ${ }^{23}$ along with the same functional and basis sets. The above quantum chemical calculations were performed using the Gaussian 03 suite. ${ }^{24}$ Charge difference density ${ }^{17}$ was employed to visualize intracluster excitation or charge transfer (CT) between pyrazine and both the tip and the surface in electronic transitions.

The 3D-FDTD method was used for the theoretical simulation of the local electric field between tip and surface. The FDTD method is a powerful computational technique which is widely used to simulate the optical properties of plasmonic nanostructures with arbitrary sizes and shapes. ${ }^{25}$ The models of TERS and double-tip TERS can be seen from Fig. 1. In the FDTD method, the space containing the simulated model is discretized using elements called the "Yee cell". ${ }^{25}$ To accurately simulate the detailed nanostructure, a non-uniform FDTD mesh method was adopted in our calculations. This method serves to save computational resources without losing the accuracy. The number of periods of the incident sinusoidal plane wave was set to 12 to guarantee calculation convergence, which could be judged by checking whether near-zone electric field values had reached a steady state. The amplitude of the sinusoidal plane wave was set at $1 \mathrm{~V} \mathrm{~m}^{-1}$ in the calculation, and the excitation wavelengths are $1064 \mathrm{~nm}, 785 \mathrm{~nm}, 633 \mathrm{~nm}$ and $514 \mathrm{~nm}$, respectively. The angle between the tip axis and the incident light is set at $60^{\circ}$ (see Fig. 1), which is suitable according to the theoretical reports in ref. 16.

An important requirement of this method for the study of dispersive materials, such as gold or palladium, is an analytical law of dispersion. It is known that the Drude model can not accurately describe the frequency-dependent complex permittivity for metals over a wide frequency range, especially for gold, since the interband transitions play an important role in the dielectric function. In recent years, some efficient analytical models have been developed to describe the dielectric response of dispersive materials. ${ }^{26-29}$ In this work, to simulate the complex permittivity of gold and palladium, instead of the simple Drude model, we adopted the general Drude model with the form ${ }^{29,30}$

$$
\varepsilon(\omega)=\varepsilon_{\infty}+\frac{\varepsilon_{\mathrm{s}}-\varepsilon_{\infty}}{1+\mathrm{i} \omega \tau}+\frac{\sigma}{\mathrm{i} \omega \varepsilon_{0}}
$$

where $\varepsilon_{\mathrm{s}}, \varepsilon_{\infty}, \sigma, \tau$ represent static permittivity, infinite frequency permittivity, conductivity and the relaxation time, respectively. $\omega$ is the angular frequency, and $\varepsilon_{0}$ is the permittivity of free space. The four parameters, $\varepsilon_{\mathrm{s}}, \varepsilon_{\infty}, \sigma$ and $\tau$, can be adjusted by curve-fitting techniques to correctly match the complex permittivity which can be derived from the experimentally determined optical constants through the relationships $\varepsilon_{\mathrm{r}}=n^{2}-k^{2}$ and $\varepsilon_{\mathrm{i}}=2 n k$, where $\varepsilon_{\mathrm{r}}$ and $\varepsilon_{\mathrm{i}}$ are the real and imaginary parts of the dielectric function of the dispersive material, respectively. This model for the local dielectric function of metallic materials represents the optical response over a wide frequency region, especially at visible 


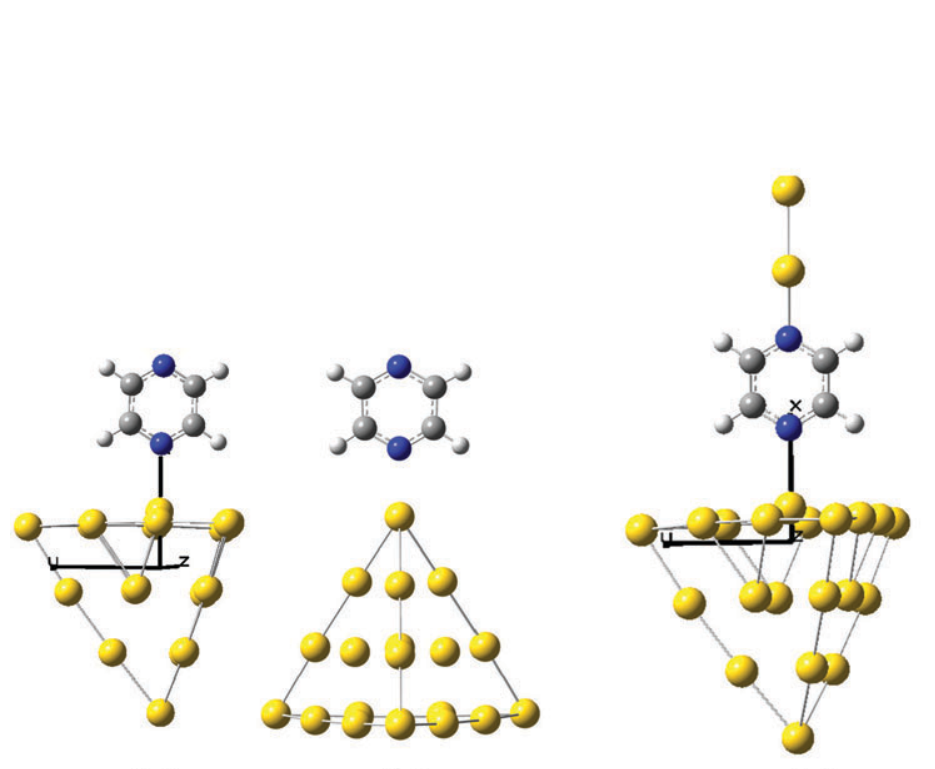

(a)

(b)

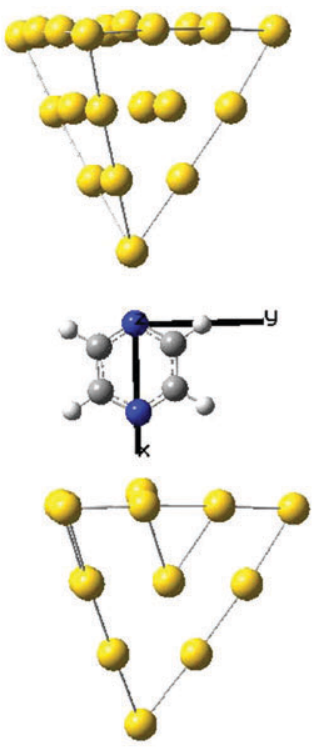

(d)

Fig. 2 (a) S-complex and (b) V-complex SERS models and (c) and (d) are TERS models for tips with different sizes.

frequencies, ensuring the accuracy of the FDTD simulation. ${ }^{29,30}$ The experimental optical constant data are taken from ref. 31 and 32 .

\section{Results and discussion}

\section{A Ground state properties}

The redistribution of the charge when forming the complex can be seen in Fig. 3. We find that, in the SERS model, $0.16 e$ is transferred from pyrazine to the surface. For the TERS model, $0.25 e$ is transferred to the surface $\left(\mathrm{Au}_{20}\right)$ and tip $\left(\mathrm{Au}_{2}\right)$, and the transferred electrons are $0.17 e$ and $0.08 e$, respectively. As the tip size increases, more electrons $(0.27 e)$ are transferred, and most of the transferred electrons $(0.17 e)$ are localized on the tip, not the surface.

Due to the interaction between pyrazine, the surface and the tip, the charge is transferred to the surface and/or tip, which results in a change in polarizability. The calculated static electronic polarizability components are listed in Table 1. It can be found that the polarizability components in TERS

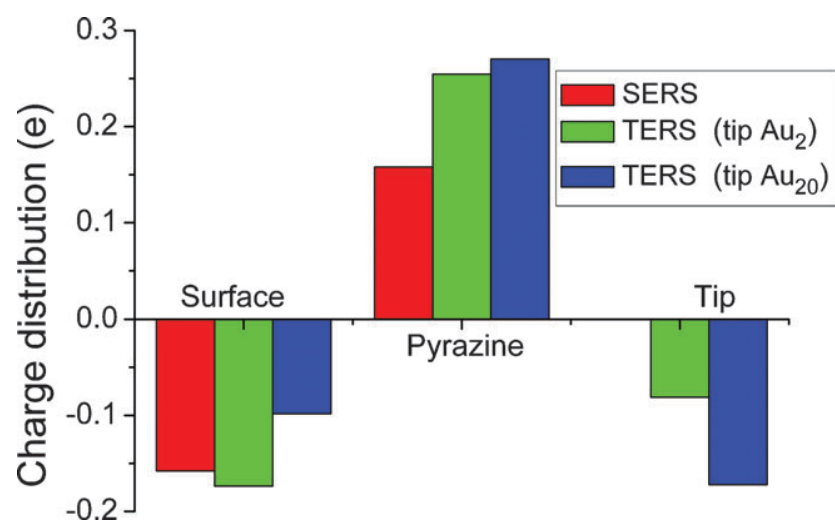

Fig. 3 Charge redistribution for the three models at the ground state. are larger than those of SERS; furthermore, with the increase in tip size, the polarizability components are increased as well. These changes in the electronic properties of the molecule will affect its NRS spectra, as shown in the simulated NRS spectra presented in Fig. 4. The intensities are enhanced by a factor of approximately 2 in the case of SERS, due to static chemical enhancement via charge transfer. The NRS spectrum of pyrazine is dominated by three intense peaks at 1041, 1273 and $1611 \mathrm{~cm}^{-1}$, which correspond to the ring-breathing, $\mathrm{C}-\mathrm{H}$ bending, and ring-breathing modes, respectively. The ringbreathing mode at $1041 \mathrm{~cm}^{-1}$ splits into two ring deformation modes for SERS, due to the lower symmetry. Additionally, these two ring deformation modes are strongly enhanced in comparison with other modes. Comparing the SERS spectrum and the TERS (tip is $\mathrm{Au}_{2}$ ) spectrum, it is clear that there are some significant changes. The two deformation modes are enhanced by factors of approximately 10 and 5, respectively. The $\mathrm{C}-\mathrm{H}$ bending mode is enhanced by a factor of approximately 20 . The ring-stretching mode at $1624 \mathrm{~cm}^{-1}$ is enhanced by a factor of approximately 140 . When the size of the tip is increased to an $\mathrm{Au}_{20}$ cluster, we find that the profile of TERS $\left(\mathrm{Au}_{2}\right.$ tip) and that of TERS $\left(\mathrm{Au}_{20}\right.$ tip) are almost the same, and all the modes were enhanced equally.

\section{B Electronic excited state properties}

We first calculated the electronic absorption spectrum of SERRS and found the first excited state to be at $\sim 578 \mathrm{~nm}$. By carefully examining the coherence of electron-hole pairs for these excited states, one can find that there are two kinds of optical electronic excitations (see Fig. 5): (a) intracluster excitation (where all the electrons and holes are localized on an $\mathrm{Au}_{20}$ cluster); (b) $\mathrm{CT}$ excitation (charge transfer from pyrazine to surface, or charge transfer from surface to pyrazine). It can be found that almost all the excited states are intracluster excitations from $578 \mathrm{~nm}$ to $425 \mathrm{~nm}$, which is direct visual evidence for the electromagnetic mechanism. 

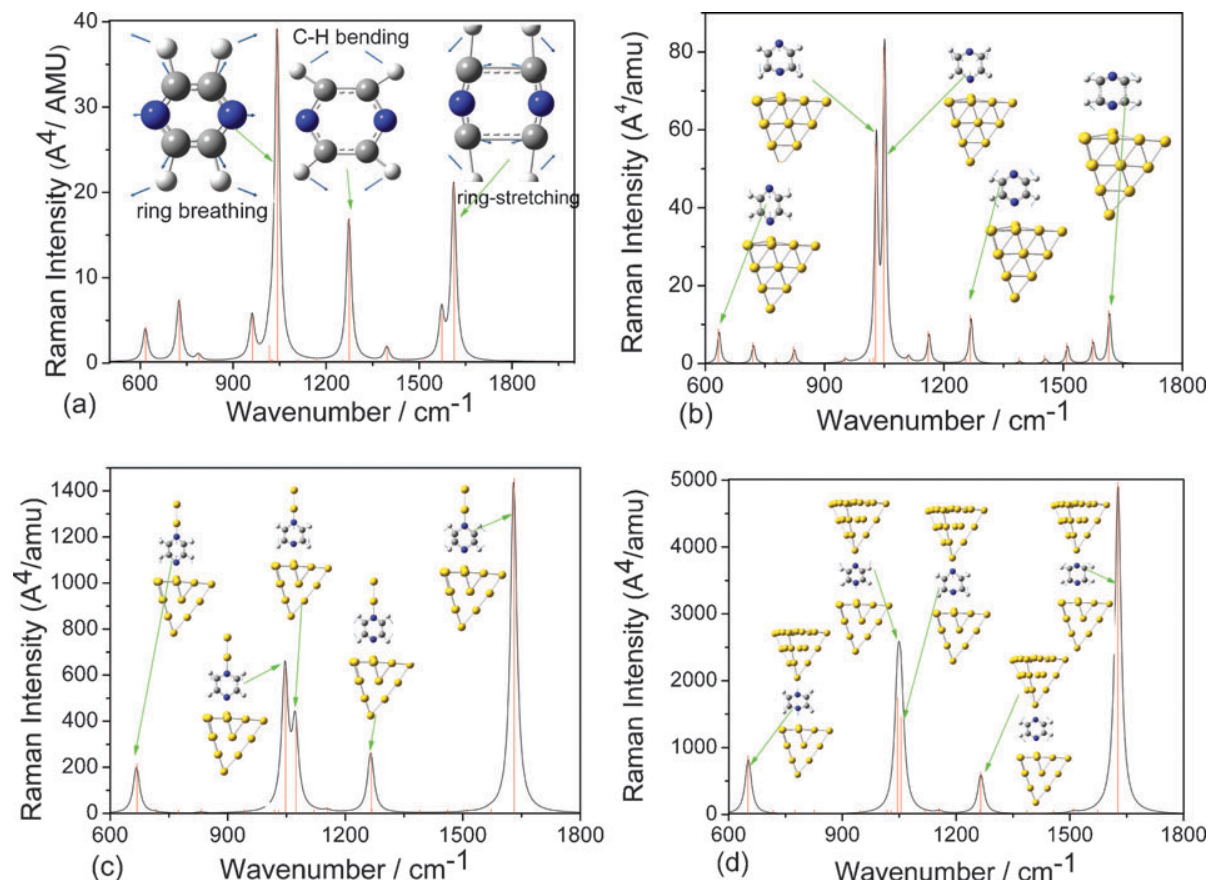

Fig. 4 (a) Normal Raman, (b) SERS and (c) and (d) TERS spectra of pyrazine for the tips with $\mathrm{Au}_{2}$ and $\mathrm{Au}_{20}$, respectively.

Table 1 Static polarizabilities (in au) for SERS and TERS models at the ground state

\begin{tabular}{lrrr}
\hline & \multicolumn{1}{c}{$x x$} & \multicolumn{1}{c}{$y y$} & \multicolumn{1}{c}{$z z$} \\
\hline Pyrazine- $\mathrm{Au}_{20}$ & 891.630 & 784.248 & 764.884 \\
$\mathrm{Au}_{2}$-pyrazine- $\mathrm{Au}_{20}$ & 1139.565 & 826.502 & 806.110 \\
$\mathrm{Au}_{20}$-pyrazine- $\mathrm{Au}_{20}$ & 2010.329 & 1481.275 & 1462.075 \\
\hline
\end{tabular}

From $435 \mathrm{~nm}$ to $375 \mathrm{~nm}$, there are a lot of CT excited states with strong oscillator strengths. This serves as direct evidence for the chemical mechanism, via CT, for TERS. Also, there are three strong intracluster excitations in this region. From $375 \mathrm{~nm}$ to $350 \mathrm{~nm}$, almost all excited states are the result of intracluster excitations. It should be noted that at $375 \mathrm{~nm}$ and $390 \mathrm{~nm}$, intracluster and CT excited states exist simultaneously, indicating that the SERRS spectrum is the result of both chemical and electromagnetic mechanisms. When the incident light is above $578 \mathrm{~nm}$, Raman spectra should result from SERS instead of SERRS, and the enhanced mechanism should be the static chemical enhancement and the electromagnetic enhancement.

Next, we calculated the electronic absorption spectrum of TERRS (see Fig. 6, where the tip is $\mathrm{Au}_{2}$ ). In this case, the first excited state is at $\sim 598 \mathrm{~nm}$, which is red shifted by $20 \mathrm{~nm}$ compared to the $S_{1}$ in SERRS, due to the influence of the tip. By carefully examining the coherence of electron-hole pairs for these excited states, one can find that there are three kinds of optical electronic excitations (see Fig. 6): (a) intracluster excitation (where all the electrons and holes are localized on the $\mathrm{Au}_{20}$ cluster); (b) $\mathrm{CT}$ excitation (charge transfer from surface and/or tip to pyrazine); (c) intercluster CT excitation (charge transfer from tip to surface).

For the $\mathrm{Au}_{20}$ cluster tip, we calculated the first ten singlet excited states in the optical absorption spectrum (see Fig. 7).

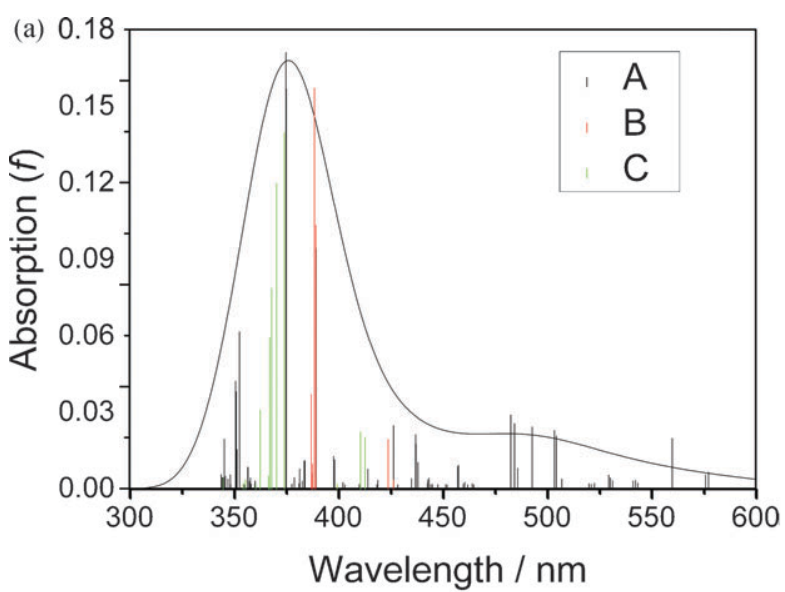

(b)

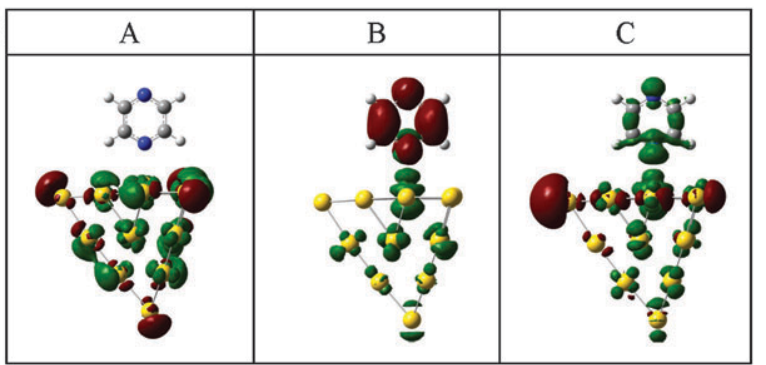

Fig. 5 (a) Optical absorption for the SERS model and (b) charge difference density for cases A, B and C in the inset in (a); the green and red stand for holes and electrons, respectively.

With the increase in tip size, the optical absorption spectrum is red shifted to $688 \mathrm{~nm}$. The charge transfer between molecule and tip (or surface), between tip and surface, and intracluster (tip or surface) charge redistributions can be found.

It should be mentioned that quantum chemical calculations require a huge computational effort, and the calculations are 
(a)

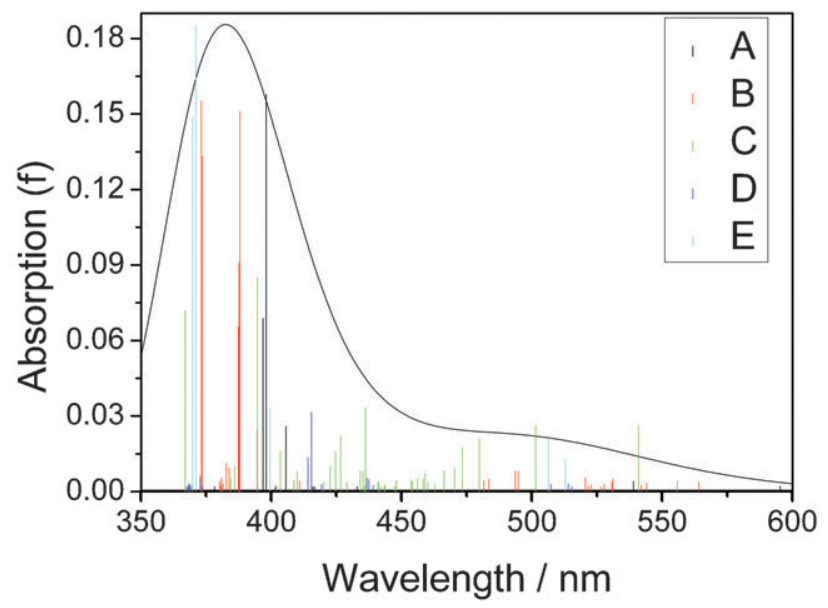

(b)

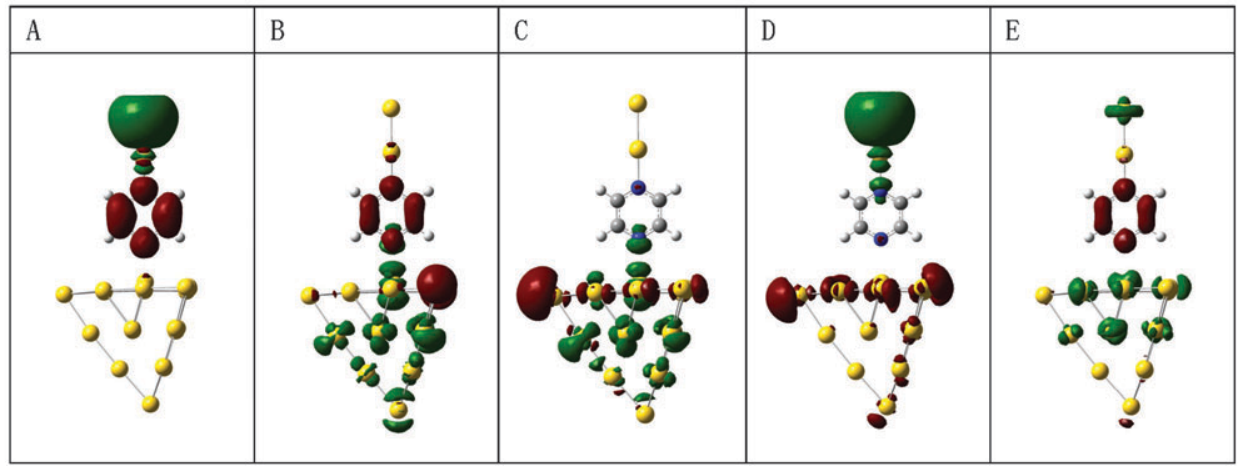

Fig. 6 (a) Optical absorption for the TERS model; there are five kinds of optical properties (for A, B, C, D and E), which are illustrated in (b), where the green and red stand for holes and electrons, respectively.

limited by computational resources. Consequently, the clusters in quantum chemical calculations may not model a bulk material very well and there might be aspects that are missing, such as imperfections in surface roughness. To better describe the optical properties of TERS in the bulk material and a real tip, we investigated electromagnetic enhancements on TERS with FDTD methods.

\section{TERS at intense electric fields}

In our quantum chemical calculations, the distance between tip and surface was not changed when the geometry of tip-molecule-surface was fully optimized. In most of our experiments, the distance between tip and surface is tunable, which will affect the TERS intensities. In this paper, the distance-dependent (between tip and surface) distributions of the near electric field enhancement for TERS at different incident lights were investigated theoretically using a 3D-FDTD method. A model consisting of a tip and surface was used in our 3D-FDTD simulation (see Fig. 1).

Fig. 8(a) gives a typical field distribution of a tip-plane system ( $y z$ plane at $x=0)$, with a $1 \mathrm{~nm}$ distance between tip and surface, illuminated with $785 \mathrm{~nm}$ light. It is found that the strongest electromagnetic enhancement is localized between tip and surface, which is usually called a "hot site". The field in the hot site is about 300 times larger than the incident light and is caused by the strong near field coupling between the tip and the plane. In a typical TERS experiment, the molecules in the hot site give the main contribution to the Raman signals. Fig. 9(a) shows local electromagnetic field enhancements ( $M=\left|\frac{E_{\mathrm{loc}}}{E_{\mathrm{in}}}\right|$, where $E_{\mathrm{loc}}$ and $E_{\mathrm{in}}$ stand for the local and incoming field, respectively) with different distances and different incident lights (the TERS enhancement is $M^{4}$ ). It is found that the local electromagnetic field enhancements decrease dramatically with increase of gap distance. This is due to the fact that the near field decays exponentially with distance from the metal surface, causing the coupling of the tip and the plane to weaken as the gap increases. At the same gap distance, the electromagnetic enhancement with incident light of $1064 \mathrm{~nm}$ is the strongest. This might be due to the fact that the tip used in this case has a longer shape and, hence, a plasmonic resonant peak that is located at a long wavelength. Additionally, the plane is not large enough in comparison with the incident wavelength. The incident angle of $30^{\circ}$ has a transverse field distribution which may cause the TERS enhancement at the incident light of $785 \mathrm{~nm}$ to be less than the TERS enhancement at the incident light of $633 \mathrm{~nm}$. However, this is beyond the scope of this article, and the simulation of the resonant peak of the system is not included here.

\section{Electromagnetic enhancement on double-tip TERS}

Fig. 8(b) gives a typical field distribution of a double tip system ( $y z$ plane at $x=0$ ) with a distance of $1 \mathrm{~nm}$ between 


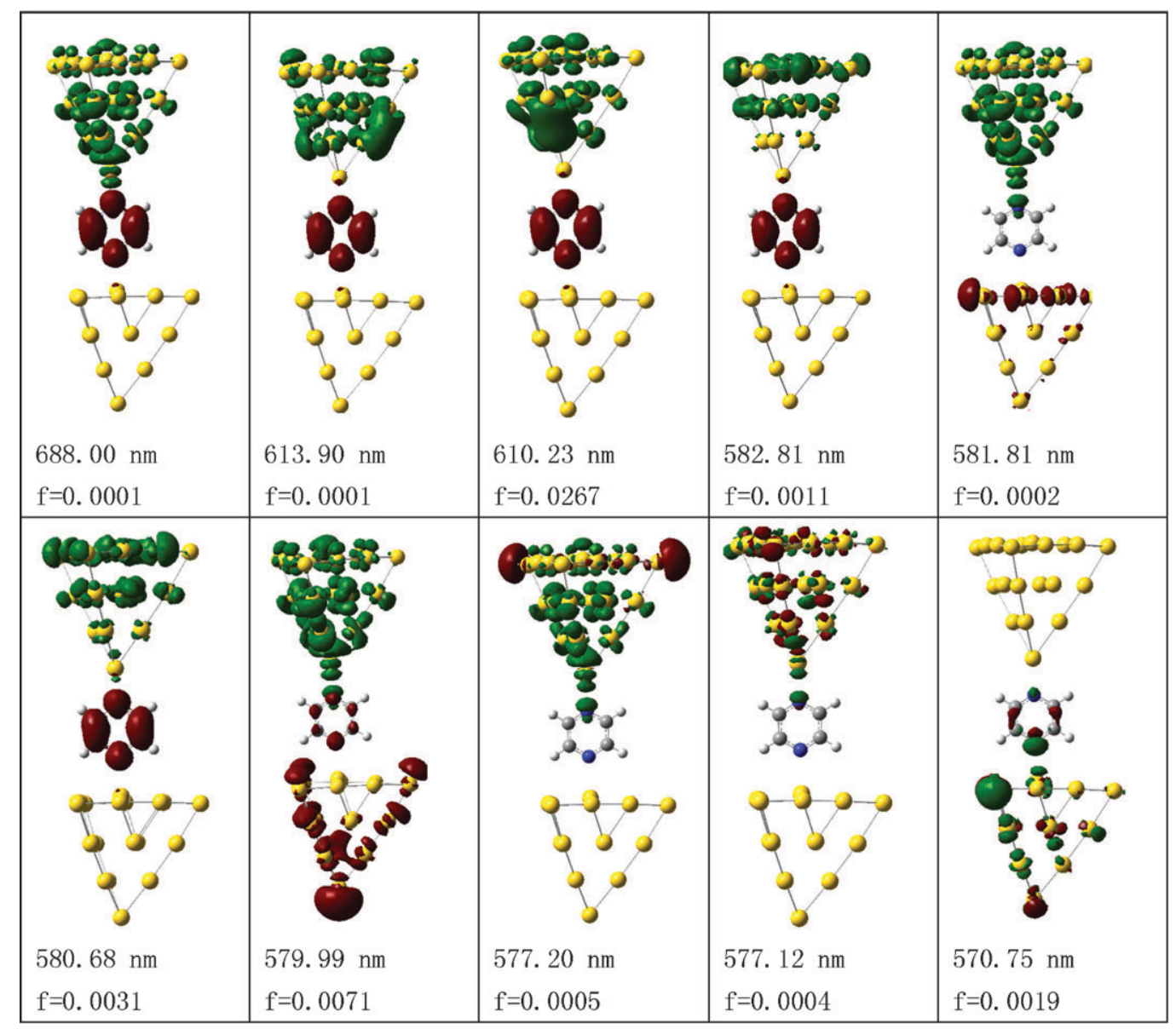

Fig. 7 Optical absorption for the TERS model, where the green and red stand for holes and electrons, respectively.

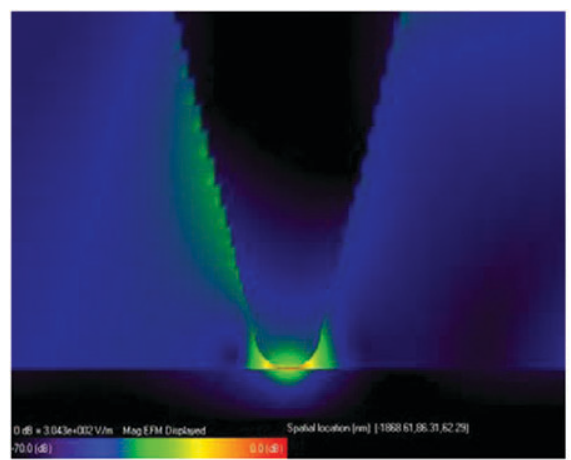

(a)

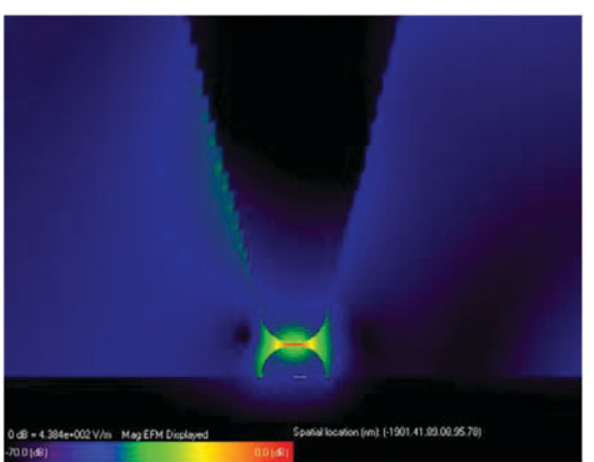

(b)

Fig. 8 Near field $E$ distribution in tip-plane (a) and double-tip (b) systems, with a $1 \mathrm{~nm}$ gap between tip and surface/nanoparticle, excited by $785 \mathrm{~nm}$ laser.

tip and nanoparticle using an incident light of $785 \mathrm{~nm}$. As expected, the strongest electromagnetic enhancement is localized between tips. Fig. 9(b) shows local electromagnetic field enhancements $(M)$ at different distances and different incident lights. It is also found that the local electromagnetic field enhancements decrease dramatically with the increase of gap distance due to the gradual weakening of the coupling between the two tips with increasing gap distance. However, the field excited by $633 \mathrm{~nm}$ light decreases more slowly than the other two. This is attributed to the blue-shift of the plasmon resonant peak due to the increase in gap distance. $^{33}$

Fig. 10(a)-(c) shows the enhancement $\left(M^{4}\right)$ of double-tip TERS with incident light of $633 \mathrm{~nm}, 785 \mathrm{~nm}$ and $1064 \mathrm{~nm}$, respectively. Obviously, the enhancement of the double tip is much stronger than that of conventional tip-plane TERS systems. This has been demonstrated experimentally by Chen and coworkers in ref. 7. Physically, a nanosphere on a perfect 

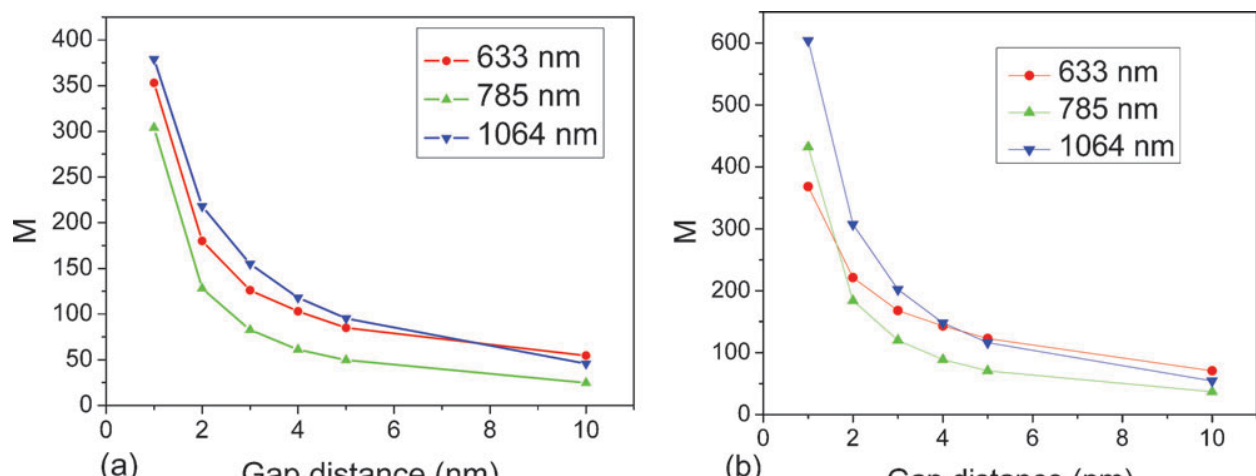

(b) Gap distance (nm)

Fig. 9 (a) Local $E$ field enhancement $\left(M=\mid \frac{E_{\text {loc }}}{E_{\text {in }}}\right)$ for conventional TERS with different gaps, at different incident lights and (b) $M=\left|\frac{E_{\text {loc }}}{E_{\text {in }}}\right|$ for double-tip TERS with different gaps, at different incident lights.

conductor with a $1 \mathrm{~nm}$ gap is equivalent to two nanospheres with a $2 \mathrm{~nm}$ gap, which means that the tip-plane system with a golden substrate and $1 \mathrm{~nm}$ gap has a smaller enhancement factor of the electromagnetic field than two nanospheres with a $2 \mathrm{~nm}$ gap, and consequently smaller than a double tip system with a $1 \mathrm{~nm}$ gap which can be treated as two nanospheres separated by a gap of approximately $1 \mathrm{~nm}$. The Au surface in a TERS experiment is not a perfect conductor in ref. 7. The local electromagnetic field enhancements decrease dramatically with the increase of gap distance. Also, at the same gap distance, the strongest enhancement of the double-tip TERS is at the incident light of $1064 \mathrm{~nm}$.

The ratio of TERS enhancements between double-tip TERS and conventional TERS with different gap distances and at the incident lights of $633 \mathrm{~nm}, 785 \mathrm{~nm}$ and $1064 \mathrm{~nm}$ can be seen from Fig. 11. We define the ratio as $R=\left(\frac{M_{\mathrm{D}}}{M_{\mathrm{S}}}\right)^{4}$, where D and $S$ stand for double-tip TERS and conventional TERS, respectively. The largest ratio of TERS enhancement is $R=4.3$ at the incident light of $632.8 \mathrm{~nm}$, when the gap distance is $5 \mathrm{~nm}$. From $1.0 \mathrm{~nm}$ to $5.0 \mathrm{~nm}$, the ratio of TERS enhancement is increased with the increase of the gap distance at the incident light of $632.8 \mathrm{~nm}$. At a suitable experimental distance (around $2 \mathrm{~nm}$ ), $R$ is about 2. At the incident light of $785 \mathrm{~nm}, R$ is almost constant with the increase in the gap distance from $1.0 \mathrm{~nm}$ to $5.0 \mathrm{~nm}$, and $R \approx 4$. When the incident light is $1064 \mathrm{~nm}, R$ decreases exponentially, and the largest $R$ is 6.4 when the gap distance is $1 \mathrm{~nm}$. Hence, the double-tip TERS is the most important for the situation in which incident light of $1064 \mathrm{~nm}$ is used. Note that we also increase the length of the tip to $525 \mathrm{~nm}$ from $325 \mathrm{~nm}$ for the case of $1064 \mathrm{~nm}$ incident light, when the gap distance is $1 \mathrm{~nm}$. The TERS enhancements are $5.31 \times 10^{10}$ and $1.05 \times 10^{10}$ for double-tip TERS and conventional TERS, respectively, and $R=5.1$, which shows that the results (tip length is $325 \mathrm{~nm}$ ) are reasonable. Consequently, when the gap size is around $1 \mathrm{~nm}, R_{\left(\lambda_{\text {in }}=1064 \mathrm{~nm}\right)}>R_{\left(\lambda_{\text {in }}=785 \mathrm{~nm}\right)}>R_{\left(\lambda_{\text {in }}=633 \mathrm{~nm}\right)}$, where $\lambda_{\text {in }}$ is the incident light wavelength. When the distance of the gap is around $2 \mathrm{~nm}, R_{\left(\lambda_{\mathrm{in}}=1064 \mathrm{~nm}\right)} \approx R_{\left(\lambda_{\mathrm{in}}=785 \mathrm{~nm}\right)}>R_{\left(\lambda_{\mathrm{in}}=633 \mathrm{~nm}\right)}$.

The coupling of tip and surface may be interpreted with plasmon interactions, which depend strongly on the gap distance between them. Similar to the particle-film system, ${ }^{34,35}$ the resonance wavelengths will shift to the red when the separation decreases, due to dipole-dipole interactions. These shifts of the
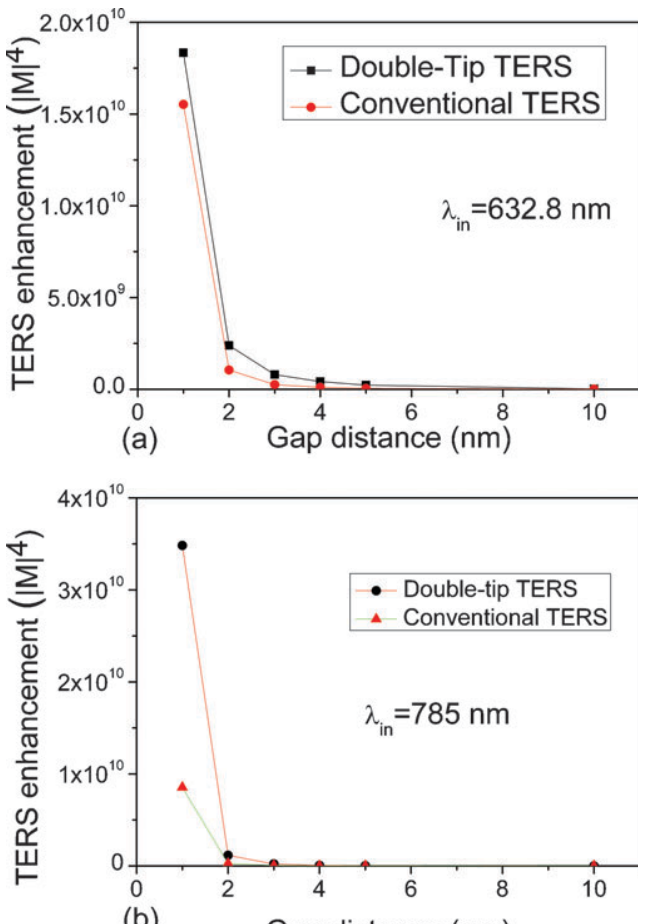

(b) Gap distance (nm)

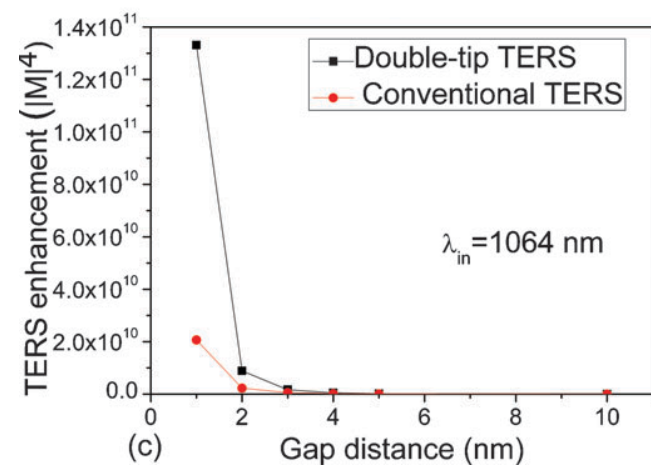

Fig. 10 TERS enhancements for the double-tip TERS and conventional TERS at the incident lights of (a) $632.8 \mathrm{~nm}$, (b) $785 \mathrm{~nm}$ and (c) $1064 \mathrm{~nm}$.

resonant peaks result in the complicated dependence of $R$ on separation at the special frequencies as shown in Fig. 11. 


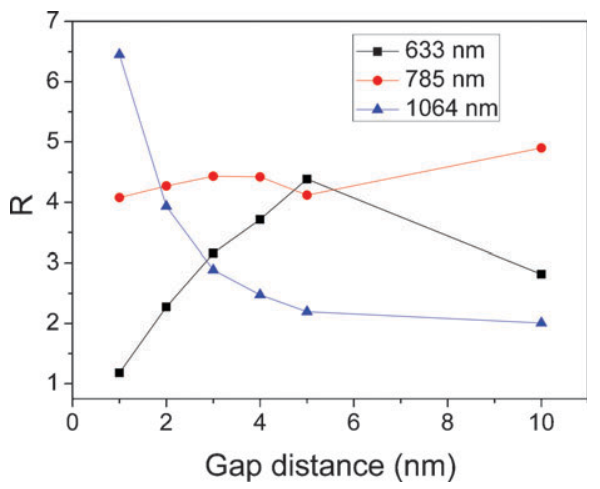

Fig. $11 \quad R=\left(\frac{M_{\mathrm{D}}}{M_{\mathrm{S}}}\right)^{4}$ with different gap distances and at the incident lights of $633 \mathrm{~nm}, 785 \mathrm{~nm}$ and $1064 \mathrm{~nm}$, where D and S stand for double-tip TERS and conventional TERS, respectively.

\section{Conclusion}

The nature of the chemical enhancement in TERS was revealed visually with charge difference density. There are four kinds of charge transfer: (1) tip to molecule, (2) surface to molecule, (3) tip and surface to molecule simultaneously, and (4) tunneling charge transfer between tip and surface. Intracluster (tip or surface) charge redistribution visually reveals direct evidence for the electromagnetic mechanism. The distance (between tip and surface) dependent distribution of the near electric field enhancement for TERS at different incident lights was also obtained theoretically through calculations employing the 3D-FDTD method. The TERS enhancement decreases dramatically as the distance between tip and substrate is increased. Electromagnetic enhancement of the double-tip TERS is less than 10 times that of conventional TERS.

\section{Acknowledgements}

This work was supported by the National Natural Science Foundation of China (Grant No. 10874234, 20703064, and 10625418), the National Basic Research Project of China (Grant No. 2009CB930701, 2009CB930703 and 2007CB936804), the Sino-Swedish Collaborations about Nanophotonics and Nanoelectronics (2006DFB02020), the Natural Science Foundation of Fujian Province of China (No. E0710028), and the "Bairen" projects of CAS.

\section{References}

1 H. X. Xu, E. J. Bjerneld, M. Kall and L. Borjesson, Phys. Rev. Lett., 1999, 83, 4357.

2 A. Otto, H. Grabhorn and W. Akemann, J. Phys.: Condens. Matter, 1992, 4, 1143.

3 M. A. Yong, J. A. Dieringer and R. P. Van Duyne, in Tip Enhancement, ed. S. Kawata and V. M. Shalaev, Elsevier, Amsterdam, 2007, ch. 1.

4 R. M. Stöckle, Y. D. Suh, V. Deckert and R. Zenobi, Chem. Phys. Lett., 2000, 318, 131.

5 B. Pettinger, B. Ren, G. Picardi, R. Schuster and G. Ertl, Phys. Rev. Lett., 2004, 92, 096101; K. F. Domke, D. Zhang and B. Pettinger, J. Am. Chem. Soc., 2006, 128, 14721.
6 H. Watanabe, Y. Ishida, N. Hayazawa, Y. Inouye and S. Kawata, Phys. Rev. B: Condens. Matter Mater. Phys., 2004, 69, 155418.

7 J. N. Chen, W. S. Yang, K. Dick, K. Deppert, H. Q. Xu, L. Samuelson and H. X. Xu, Appl. Phys. Lett., 2008, 92, 093110.

8 W. Zhang, X. Cui, B. Yeo, T. Schmid, C. Hafner and R. Zenobi, Nano Lett., 2007, 7, 1401.

9 Y. Inouye, P. Verma, T. Ichimura and S. Kawata, in Tip Enhancement, ed. S. Kawata and V. M. Shalaev, Elsevier, Amsterdam, 2007, ch. 3.

10 M. Moskovits, Rev. Mod. Phys., 1985, 57, 783.

11 K. Kneipp, H. Kneipp, I. Itzkan, R. R. Dasari and M. S. Feld, Chem. Rev., 1999, 99, 2957.

12 J. R. Lombardi, R. L. Birke, T. H. Lu and J. Xu, J. Chem. Phys., 1986, 84, 4174.

13 L. L. Zhao, L. Jensen and G. C. Schatz, J. Am. Chem. Soc., 2006, 128, 2911.

14 M. T. Sun, S. S. Liu, M. D. Chen and H. X. Xu, J. Raman Spectrosc., 2009, 40, 137.

15 A. L. Demming, F. Festy and D. Richards, J. Chem. Phys., 2005, 122, 184716; H. Furukawa and S. Kawata, Opt. Commun., 1998, $148,221$.

16 Z. L. Yang, J. Aizpurua and H. X. Xu, J. Raman Spectrosc., 2009, DOI: $10.1002 /$ jrs. 2429.

17 M. T. Sun, S. B. Wan, Y. J. Liu, J. Yu and H. X. Xu, J. Raman Spectrosc., 2008, 39, 402

18 J. Li, X. Li, H. J. Zhai and L. S. Wang, Science, 2003, 299, 864.

19 P. Hohenberg and W. Kohn, Phys. Rev., 1964, 136, B864.

20 A. D. Becke, Phys. Rev. A: At., Mol., Opt. Phys., 1988, 38, 3098.

21 J. P. Perdew, J. A. Chevary, S. H. Vosko, K. A. Jackson, M. R. Pederson, D. J. Singh and C. Fiolhais, Phys. Rev. B: Condens. Matter Mater. Phys., 1992, 46, 6671.

22 P. J. Hay and W. R. Wadt, J. Chem. Phys., 1985, 82, 270.

23 E. K. U. Gross and W. Kohn, Phys. Rev. Lett., 1985, 55, 2850.

24 M. J. Frisch, G. W. Trucks, H. B. Schlegel, G. E. Scuseria, M. A. Robb, J. R. Cheeseman, J. A. Montgomery, Jr., T. Vreven, K. N. Kudin, J. C. Burant, J. M. Millam, S. S. Iyengar, J. Tomasi, V. Barone, B. Mennucci, M. Cossi, G. Scalmani, N. Rega, G. A. Petersson, H. Nakatsuji, M. Hada, M. Ehara, K. Toyota, R. Fukuda, J. Hasegawa, M. Ishida, T. Nakajima, Y. Honda, O. Kitao, H. Nakai, M. Klene, X. Li, J. E. Knox, H. P. Hratchian, J. B. Cross, V. Bakken, C. Adamo, J. Jaramillo, R. Gomperts, R. E. Stratmann, O. Yazyev, A. J. Austin, R. Cammi, C. Pomelli, J. Ochterski, P. Y. Ayala, K. Morokuma, G. A. Voth, P. Salvador, J. J. Dannenberg, V. G. Zakrzewski, S. Dapprich, A. D. Daniels, M. C. Strain, O. Farkas, D. K. Malick, A. D. Rabuck, K. Raghavachari, J. B. Foresman, J. V. Ortiz, Q. Cui, A. G. Baboul, S. Clifford, J. Cioslowski, B. B. Stefanov, G. Liu, A. Liashenko, P. Piskorz, I. Komaromi, R. L. Martin, D. J. Fox, T. Keith, M. A. Al-Laham, C. Y. Peng, A. Nanayakkara, M. Challacombe, P. M. W. Gill, B. G. Johnson, W. Chen, M. W. Wong, C. Gonzalez and J. A. Pople, GAUSSIAN 03 (Revision E.1), Gaussian, Inc., Wallingford, CT, 2004.

25 K. S. Kunz and R. J. Luebber, The Finite Difference Time Domain Method for Electromagnetics, CRC Press, New York, 1993.

26 A. Vial and T. Laroche, J. Phys. D: Appl. Phys., 2007, 40, 7152.

27 P. G. Etchegoin, E. C. Le Ru and M. Meyer, J. Chem. Phys., 2006, 125, 164705 .

28 F. Hao and P. Nordlander, Chem. Phys. Lett., 2007, 446, 115.

29 J. T. Krug II, E. J. Sanchez and X. S. Xie, J. Chem. Phys., 2002, 116, 10895.

30 Z. Q. Tian, Z. L. Yang, B. Ren and D. Y. Wu, Top. Appl. Phys., 2006, 103, 125.

31 P. B. Johnson and R. W. Christy, Phys. Rev. B: Condens. Matter Mater. Phys., 1972, 6, 4370.

32 Handbook of Optical Constants of Solids, ed. E. D. Palik, Academic press, New York, 1985.

33 J. Renger, S. Grafström, L. M. Eng and V. Deckert, J. Opt. Soc. Am. A, 2004, 21, 1362.

34 G. Lévêque and O. J. F. Martin, Opt. Express, 2006, 14, 9971.

35 F. Le, N. Z. Lwin, J. M. Steele, M. Kall, N. J. Halas and P. Nordlander, Nano Lett., 2005, 5, 2009. 